\title{
Photometric observations of the mutual phenomena of the Galilean Satellites of Jupiter in 1997 and 2003 at the Royal Observatory of Belgium ${ }^{\star} \star \star \star$
}

\author{
T. Pauwels ${ }^{1}$, P. Vingerhoets ${ }^{2}$, and J. Cuypers ${ }^{1}$ \\ 1 Koninklijke Sterrenwacht van België, Ringlaan 3, 1180 Brussel, Belgium \\ e-mail: Thierry.Pauwels@oma.be \\ 2 Vereniging Voor Sterrenkunde, Werkgroep Bedekkingen, Groenstraat 12, 2640 Mortsel, Belgium
}

Received 2 July 2004 / Accepted 16 February 2005

\begin{abstract}
In this paper we describe the observations of the mutual phenomena of the Galilean Satellites of Jupiter performed at the Royal Observatory of Belgium, as part of the PHEMU97 and PHEMU03 campaigns. The paper describes the observational technique, the data reduction and summarises the results obtained. The tables with the data are available at the CDS.
\end{abstract}

Key words. methods: data analysis - methods: observational - eclipses - occultations planets and satellites: individual: Jovian Galilean Satellites

\section{Introduction}

Eclipses, occultations and transits of the Galilean satellites are well-known phenomena. In each of these phenomena, Jupiter and one of its satellites are involved. Less known are the mutual phenomena of the satellites where one satellite eclipses or occults another one. These phenomena occur only when the Earth (for the occultations) or the Sun (for the eclipses) is nearly in the orbital plane of the satellites. Thus, these phenomena take place only during "seasons", which last about one year and reoccur about every six years.

The interest in observing the mutual phenomena is to better determine the positions of the satellites. The accuracy in position that can be achieved by analysing photometric observations of the satellites involved in a mutual event is much better than direct astrometric measurement, and also much better than what could be achieved by observing normal events (involving Jupiter), since the light curve obtained from a mutual event is not affected by a complex atmosphere, as is the case with a light curve obtained from an event involving Jupiter. Under the best conditions, and taking into account the surface features of the satellite (especially when the occulted or eclipsed satellite is Io), a positional precision of 8 kilometers can be achieved (Arlot 1997).

$\star$ Tables 3-22 are only available in electronic form at the CDS via anonymous ftp to cdsarc.u-strasbg. fr(130.79.125.5) or via http://cdsweb.u-strasbg.fr/cgi-bin/qcat?]/A+A/437/705

$\star \star$ Figures 2-21 are only available in electronic form at http://www.edpsciences.org
Therefore, since 1973, at each passage of the Earth and the Sun through the orbital plane of Jupiter's satellites, there have been campaigns to observe the mutual events of the Galilean Satellites. Arlot (1997) gives more details about previous campaigns, and Aksnes (1997) gives details about the reduction of the observations.

The Royal Observatory of Belgium at Ukkel took part in both the campaigns, PHEMU97 (the events of the 1997 season) and PHEMU03 (the events of the 2003 season). In 1997 a series of problems had to be solved before we could start to observe. Therefore, the first event observed was only in September 1997, while the last observable event was in November 1997. Since in 1997 Jupiter was at a declination of $-18^{\circ}$ only a limited number of events could be observed from Ukkel, at a northern latitude of almost $51^{\circ}$, and all events were low in the sky, so that the noise in the light curves obtained is mostly due to seeing effects. In 2003, we could learn from the problems encountered in 1997, and we adjusted our observing strategy on a number of points. Since Jupiter was then at a declination of $+18^{\circ}$, more events were observable, and we could make a more severe selection, observing only those events that would give the best results.

\section{The observations}

\subsection{The telescope and the detector}

For the observations we used the Ukkel Schmidt telescope (main mirror of $1.20 \mathrm{~m}$, focal length of $2.12 \mathrm{~m}$, corrector plate of $0.85 \mathrm{~m}$ ), equipped with a Princeton CCD camera 
with a KODAK-chip in the prime focus. The chip contains $2048 \times 3072$ pixels of $9 \mu \mathrm{m} \times 9 \mu \mathrm{m}$. This corresponds to a pixel size of $0.9^{\prime \prime} \times 0.9^{\prime \prime}$, and a field of view of $30^{\prime} \times 45^{\prime}$. This chip can be read out at $430 \mathrm{kHz}$ or $1 \mathrm{MHz}$. This telescopecamera combination is not ideal for doing fast photometry, but the camera had been bought with the main intention of doing astrometric observations of minor planets. In order to obtain useful light curves of PHEMU phenomena, we had to set up special observing techniques.

\subsection{Reading out the $C C D$}

In order to get the required time resolution and accuracy of $0.1 \mathrm{~s}$ and to reduce the amount of data, the chip was defined to contain only 16 rows, rather than the 2048 real ones, with the camera shifting in $y$ only 16 lines after each exposure. In $x$ we defined windows around both the programme satellite and the reference object. In most cases this reference object was another satellite of Jupiter. In the PHEMU97 campaign these windows were defined to be 31 pixels wide, but this turned out to be a bit small. Apart from the risk of the satellite moving out of the window (because of the motion of the satellite or because of guiding errors), in many cases there was not enough sky background left to set up a reliable model of the sky background. Therefore, in the PHEMU03 campaign we defined the windows to be 60 pixels wide, leaving more sky background pixels for the modelling. The drawback of these larger windows is that it was more difficult to keep Jupiter out of the windows, or not too close. If Jupiter was close to the window, the binning of the pixels just outside of the windows (for faster readout of pixels not to be saved) could cause blooming into the window. To avoid saturation we did not use any binning. The complete sequence for a phenomenon contains some 20000 to 30000 images, which were acquired with the shutter remaining continuously open, also during readout.

\subsection{The reference object}

In order to be as independent as possible of variations in sky transparency (this was expected to be very variable during the observation, due to the variable weather conditions and in 1997 due to the low altitude of Jupiter), a reference object had to be monitored at the same time as the satellite(s) involved in the phenomenon. Usually this was another satellite of Jupiter.

However, in one case (in 1997), no other satellite could be used. There was a 6th magnitude star slightly less than 30 arcmin from Jupiter, so that Jupiter and the star could just be positioned on the same frame, and this star was used as a reference, but the distance was too large, so that the correlation in sky transparency between the satellite and the reference star was very poor.

In one other case (in 2003), there were two phenomena too close to each other to redefine new windows and generate time flashes (see Sect. 2.5) between the phenomena. In that case, there was only one possibility, monitoring both satellites involved in a phenomenon at the same time, and using the satellite involved in the second phenomenon as a reference object for the first phenomenon and vice versa.

\subsection{The filter}

Some of the phenomena took place very close to Jupiter. Since Jupiter is very bright, one could expect a significant sky background that would not be constant over the image. In order to reduce this sky background we purchased a special-purpose filter, with a half width of about $20 \mathrm{~nm}$, centred at $892 \mathrm{~nm}$, the methane band, in which Jupiter is very dark. However, this filter also reduces the brightness of the satellites, and hence also the signal-to-noise ratio. For some of the phenomena in 1997, when Jupiter was too low in the sky, or when the sky was too nebulous, this filter would have absorbed too much light and too little signal would have been left to do photometry. Therefore, for each phenomenon individually we decided whether to use the filter or not, depending on the distance between the satellites and Jupiter, the elevation of Jupiter above the horizon and the quality of the sky. In 2003 we only observed phenomena high enough in the sky, so that we could use the filter systsematically for all phenomena.

If no filter is used, the response of the CCD-camera is close to the $R$ band.

\subsection{The timing of the images}

Getting an accurate timing was a special problem. The only way to get the required accuracy was to put time marks on the images themselves. This was achieved by generating light flashes at the exact minute UTC. The increased background of some of the images gives a timing of the image with a precision of $0.1 \mathrm{~s}$.

In the few cases where the flashing mechanism failed, timings were recorded by hand. With a test during the day, when timings were recorded both ways, we found that this method could also give a precision of $0.1 \mathrm{~s}$.

\subsection{Selecting the phenomena}

During the PHEMU97 campaign, due to its declination the maximum elevation that Jupiter could reach in the Ukkel sky was $21 \mathrm{deg}$. Since the Ukkel sky degrades very rapidly with decreasing elevation, we observed only phenomena not too far from the meridian (although 199709293 ECL 2 was observed at only 12 deg altitude). We also excluded phenomena taking place too close to Jupiter. However, we did not place a constraint on the flux drop.

Since Jupiter was at higher declination during the PHEMU03 campaign than was the case for the PHEMU97 campaign, there were more observable phenomena from Ukkel and we could be more severe in the criteria for selecting the phenomena. As a rule, we wanted to have Jupiter at least $40 \mathrm{deg}$ above horizon (with 200302171 OCC 2 at only 32 deg elevation), the sun at least 9 deg below horizon, the phenomenon taking place far enough from Jupiter (1.3 Jupiter radii turned out to be too close), and the flux drop deep enough to be measurable. 


\section{The reductions}

\subsection{Dark frames and flat fields}

For each observation, specific windows were defined around the satellites involved. The position of the windows was dependent on the position of the satellites and their relative motion. This means that the dark current and the flat fielding of the sky background were different for each observation. A dark frame and a flat field was taken for each observation in exactly the same way as the phenomenon observed, except that they were restricted to only 1000 images. Since for each image of the same observation the dark current and the flat fielding is considered to be the same, both were averaged over all the frames, producing one dark frame and one flat field of $62 \times 16$ pixels or $120 \times 16$ pixels each. The satellites, however, were not spread over many pixels, but illuminated a few particular pixels on the chip. Therefore, for flat fielding the satellites, a normal flat field (a full image of $3072 \times 2048$ pixels) had to be used and we had to know on which pixel exactly the satellite was positioned.

\subsection{Location of the satellite}

To find the position of the satellite on the CCD, one has to find on which image the satellite first appears. This is necessary to flat field the image of the satellite and to set up the timing of each image. If clouds caused the satellite to disappear, or if the first image of the satellite is not visible, one has to analyse the whole sequence or full images taken before and/or after the event to compute the drift of the telescope, and deduce from it the number of missing images to retrieve the position of the satellite on the chip.

\subsection{Timing}

For the timing, the average sky background was computed for each image. A sudden increase in the sky background corresponds to a flash. This way, it was found that the frequency of images was 610.77 images per minute for the PHEMU97 campaign and 534.55 images per minute for the PHEMU03 campaign (new software on the PC explains the difference in acquisition speed). Moreover, this acquisition speed turned out to be very stable.

By identifying the image number of images with increased sky background, and using the corresponding minute, a linear transformation could be established between image number and time. For the timing of the satellite images, the count of the satellite images had to be used.

\subsection{Modeling the sky background}

Modeling the sky background turned out to be the most difficult part of the reduction in the PHEMU97 campaign. Clearly, improvements could be made, and therefore the approach in the PHEMU03 campaign was rather different from the one used in the PHEMU97 campaign.

In the PHEMU97 campaign we had defined windows of no more than $31 \times 16$ pixels around each satellite, while a satellite image was assumed to occupy $16 \times 16$ pixels on the image. If the satellite is well centred on the window, there are $7 \times 16$ pixels left on both sides to model the sky background. However, centering the satellite is not always possible. First, there are some imperfections in the guiding of the telescope as well as the effect of the seeing. Second, during the observation (up to $40 \mathrm{~min}$ ) the relative distance of the satellites changed, while the relative distance between the two windows could not be modified. Therefore, the relative distance between the two windows was chosen to be the relative distance of the two satellites at the maximum of the phenomenon. This often causes the satellites to lie along the border of the windows at the beginning and the end of the phenomenon, leaving only very few pixels on one side to model the sky background. Third, keeping the satellite at the right place was not easy. As a consequence sometimes a satellite drifted to the border of the window.

To model the sky background a different approach had to be taken, depending on whether the observation had been done with the methane filter or not. If the filter had been used, the quantity of sky background was low and smooth enough to assume that it was linear in $x$ (linear in the distance to Jupiter) and constant in $y$ (the direction more or less perpendicular to Jupiter). During the observation, however, there were very large variations in the quantity of sky background and the transparency of the sky. This was due both to the rapidly varying weather conditions and the low altitude of Jupiter (usually between 15 and $20 \mathrm{deg}$ ). Therefore, we fitted a polynomial, linear in $x$, constant in $y$, and third degree in the image number to 150 successive images, and applied this model the central 50 images of these 150 . This process was repeated iteratively each time with a shift of 50 images, until the whole film had been processed. In a few cases it turned out that a linear model in $x$ was not adequate to model the sky background, and in that case we switched to a second degree polynomial. To model the sky background, the $x$-coordinate is referred to a coordinate system that is fixed to the satellite (which can move on the chip) and not to the CCD. This is because we assume that the sky background is dependent on the distance to Jupiter, and that the satellite-Jupiter distance may be considered to remain constant over 150 images (about $14 \mathrm{~s}$ ), whereas due to guiding corrections, the satellite can move considerably on the chip.

If no filter had been used, the sky background was highly non-linear in $x$. Therefore we used by default a third-degree polynominal to fit the sky background in $x$. The approach in $y$ and in the image number remained the same as in the case of an observation with the filter. In some cases it turned out to be very difficult to model the sky background when the satellite got too close to the border. Not enough pixels of sky background were left on that side to fit a third degree polynomial, causing the model of the sky background to suffer from bad extrapolation. In those cases a second degree polynomial fit was tried, and if that did not help, as a last resort the box surrounding the satellite was reduced from $16 \times 16$ pixels to $12 \times 16$ pixels, leaving more sky background. If that did not help either, the portion of the film in which the sky background could not be modeled was removed from the final light curve.

Learning from the mistakes in the PHEMU97 campaign, to model the sky background, we followed much better 
procedures in the PHEMU03 campaign. First, we used windows 60 pixels wide, such that there is enough sky background left in the image after masking the image of the satellites. Second, since the observed phenomena were high in the sky and produced enough signal, such that we could use the methane band filter for all phenomena, we could use a linear model in $x$ for all events. Third, rather than removing a window with a fixed size around each satellite, we used software automatically determining for each pixel whether it contained sky background or an object. The advantage of this method is that it will remove no more pixels than strictly necessary around each satellite, but it will also efficiently remove stars that may appear on the frames, Jupiter or blooming by Jupiter that sometimes is visible, as well as effects produced by cosmic rays. Thus a maximum of useful pixels with sky background is left, and modeling the sky background this way never failed.

Images affected by flashes for time marks could not be flat fielded and were removed from the final light curve.

\subsection{Photometry}

The final photometry was obtained by summing the flux over the whole region containing a satellite. No attempt was made to fit a particular PSF (point spread function). This would have failed anyway, because of seeing effects. The shape of the images of the satellites is highly variable from one image to the next. In the PHEMU97 campaign the flux is summed over a box with a fixed size; in the PHEMU03 campaign better software determined which pixels contain the signal from the satellite, and the summing of the flux was restricted to these pixels. This approach turned out to give slightly better results in a few cases.

\section{Results and discussion}

For the PHEMU97 campaign, a film could be recorded for 12 phenomena, but only for 9 phenomena could a light curve be established. For the other 3 phenomena the satellites disappeared in the clouds.

For the PHEMU03 campaign, we obtained 12 films covering 14 phenomena ( 2 of the films contained 2 phenomena). One film could not be reduced because the satellites were too close to Jupiter and could not be separated from the glare caused by Jupiter. For one of the phenomena, there were numerous clouds, and only some portions of the light curve contain useful data.

Figure 1 gives as an example the light curve of phenomenon 200303153 ECL 4. The horizontal scale is in minutes from the predicted epoch of mid-event. The vertical scale gives the magnitude difference between the satellite and the reference satellite. The figure shows the individual data points in grey, and the running average in black. Figures for other phenomena are available online. The difference in dispersion between the PHEMU97 and PHEMU03 campaigns is striking, and is due to the difference in altitude of Jupiter in the sky, which is in turn caused by its difference in declination: $-18 \mathrm{deg}$ in 1997 and +18 deg in 2004 .

Table 1 lists the phenomena that we attempted to observe. The first three columns give the necessary data for

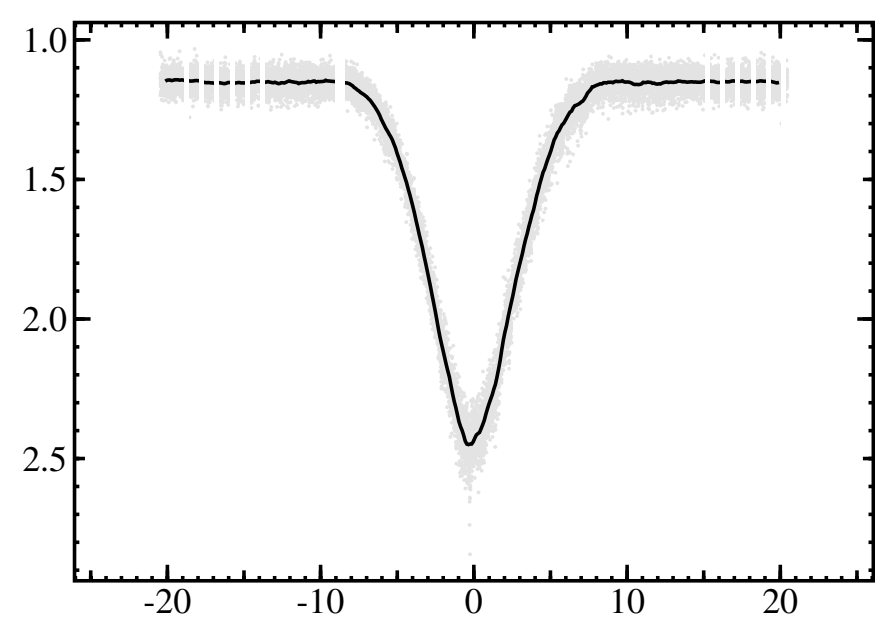

Fig. 1. The lightcurve of phenomenon 200303153 ECL 4.

identifying the phenomenon observed: date, nature of the phenomenon ("OCC" standing for "occults" and "ECL" for "eclipses", and 1, 2, 3 and 4 for Io, Europa, Ganymede and Callisto, respectively) and epoch of mid-event in TT, as announced by the IMCCE (ftp://ftp.bdl.fr/pub/ephem/ satel/phemu03/phemu03liste_eng.txt). The fourth column lists the observers, with the codes TP = Thierry Pauwels, $\mathrm{JC}=$ Jan Cuypers, PV = Piërre Vingerhoets, PL = Patricia Lampens.

The next columns summarise the results obtained. The fifth column gives the file name used for the table as available from the CDS. Identical file names means that one light curve contains two phenomena. The sixth column mentions whether we used the methane band filter, the seventh column the degree in $x$ used for modelling the sky background. Column 8 gives the satellite (in the case of an eclipse) or the satellite combination (in the case of an occultation, where during the greatest part of the phenomenon the satellites could not be separated) that we monitored, and Col. 9 the reference object, with the codes I, II, III and IV for Io, Europa, Ganymede and Callisto, the last column gives the assessment of the sky quality by the observer.

Table 2 gives notes for individual phenomena.

The detailed tables with data, one for each phenomenon, are available at the CDS and contain the following information. File names correspond to the codes given in the fifth column of Table 1. Column 1 gives the image sequence number. Missing numbers correspond to gaps in the light curve. Column 2 gives the Julian date of the epoch. Columns 3 and 4 give the magnitude of both monitored objects (the satellite involved in the phenomenon and the reference object), with an arbitrary offset, which is variable because of its dependence on the atmospheric transparency. The last column gives the magnitude difference between the programme satellite and the reference object. Usually this is the reference object minus programme satellite, but there are a few exceptions.

More detailed explanations about the method will be published in Notes Scientifiques et Techniques de l'IMCCE. 
Table 1. The list of phenomena.

\begin{tabular}{|c|c|c|c|c|c|c|c|c|c|}
\hline Date & Phenom. & $\begin{array}{c}\text { TT-mid } \\
\text { predicted }\end{array}$ & Observers & File name & $\begin{array}{l}\text { Filter } \\
\text { used? }\end{array}$ & $\begin{array}{l}\text { Deg } \\
\text { mod }\end{array}$ & $\begin{array}{l}\text { Sat } \\
\text { obs }\end{array}$ & Reference & Sky \\
\hline & & & & & PHEMU97 & & & & \\
\hline 19970918 & 1 ECL 3 & 19:35:55 & TP, PV & \multicolumn{6}{|c|}{ no lightcurve: clouds } \\
\hline 19970919 & 4 OCC 3 & $22: 15: 50$ & TP, JC, PV & 79j.dat & no & 3 & $\mathrm{III}+\mathrm{IV}$ & PPM 238231 & cirrus + cirrocumulus \\
\hline 19970921 & 3 ECL 1 & 20:32:14 & TP, JC, PV & 791.dat & yes & 1 & I & IV & light cirrus \\
\hline 19970922 & 3 ECL 2 & 19:02:59 & TP, PV & 79m.dat & yes & 1 & II & III & twilight \\
\hline 19970925 & 1 OCC 3 & $19: 55: 28$ & TP, PV & 79p1.dat & yes & 2 & I+III & II & reasonable \\
\hline 19970925 & 1 ECL 3 & $22: 33: 38$ & TP, PV & 79p2.dat & yes & 1 & III & I & reasonable \\
\hline 19970928 & 3 OCC 1 & 20:53:34 & $\mathrm{TP}, \mathrm{JC}$ & \multicolumn{6}{|c|}{ no lightcurve: clouds } \\
\hline 19970929 & 3 OCC 2 & $18: 43: 22$ & TP, PV & 79t1.dat & no & 2 & II+III & I & altocumulus + cirrocumulus \\
\hline 19970929 & 3 ECL 2 & $22: 47: 27$ & TP, PV & \multirow{2}{*}{\multicolumn{6}{|c|}{ no lightcurve: clouds }} \\
\hline 19971006 & 3 OCC 2 & $22: 13: 33$ & TP, PV & & & & no & & \\
\hline 19971007 & 4 OCC 1 & $19: 36: 46$ & $\mathrm{JC}, \mathrm{PV}$ & 7a7.dat & yes & 1 & $\mathrm{I}+\mathrm{IV}$ & III & clouds \\
\hline 19971018 & 1 OCC 2 & $18: 23: 25$ & $\mathrm{TP}, \mathrm{JC}$ & 7ai.dat & yes & 1 & I+II & III & reasonable \\
\hline 19971023 & 2 OCC 3 & 20:44:01 & $\mathrm{JC}, \mathrm{PV}$ & \multicolumn{6}{|c|}{ no lightcurve: clouds } \\
\hline 19971025 & 1 OCC 2 & 20:44:14 & $\mathrm{JC}, \mathrm{PV}$ & \multicolumn{6}{|c|}{ no lightcurve: technical problems } \\
\hline 19971118 & 3 OCC 2 & 19:10:11 & TP, JC, PV & \multirow{2}{*}{\multicolumn{6}{|c|}{$\begin{array}{l}\text { no lightcurve: clouds } \\
\text { no lightcurve: clouds }\end{array}$}} \\
\hline 19971118 & 3 ECL 1 & 19:18:48 & TP, JC, PV & & & & & & \\
\hline 19971125 & 3 OCC 1 & $18: 40: 22$ & TP, JC, PV & 7bp.dat & no & 3 & $\mathrm{I}+\mathrm{III}$ & II & cirrocumulus + altocumulus \\
\hline \multicolumn{10}{|c|}{ PHEMU03 } \\
\hline 20030109 & 3 OCC 1 & 00:44:29 & TP, PV & d18.dat & yes & 1 & I+III & II & clear sky \\
\hline 20030203 & 2 OCC 3 & $23: 31: 56$ & $\mathrm{TP}$ & d23.dat & yes & 1 & II+III & I & clear sky \\
\hline 20030203 & 2 ECL 3 & $23: 40: 40$ & TP & d23.dat & yes & 1 & II+III & I & clear sky \\
\hline 20030217 & 1 OCC 2 & $02: 51: 24$ & TP, PV & \multicolumn{6}{|c|}{ no lightcurve: too close to Jupiter } \\
\hline 20030218 & 4 ECL 3 & $20: 49: 53$ & TP, PV, JC & d2i.dat & yes & 1 & III & IV & clear sky with moon \\
\hline 20030219 & 4 OCC 1 & $20: 32: 20$ & TP, PV, JC & d2j1.dat & yes & 1 & $\mathrm{I}+\mathrm{IV}$ & III & clear sky \\
\hline 20030219 & 4 ECL 1 & $22: 16: 46$ & TP, PV, JC & d2j2.dat & yes & 1 & I & III & clear sky \\
\hline 20030227 & $1 \mathrm{OCC} 4$ & 22:04:00 & $\mathrm{JC}, \mathrm{PV}$ & d2r.dat & yes & 1 & $\mathrm{I}+\mathrm{IV}$ & III & cirrus and clouds \\
\hline 20030315 & 3 ECL 4 & $22: 15: 53$ & JC, PV & d3f.dat & yes & 1 & IV & III & clear sky \\
\hline 20030318 & 2 ECL 3 & $20: 47: 57$ & $\mathrm{JC}, \mathrm{PV}$ & d3i.dat & yes & 1 & III & IV & bad seeing_maybe some haze \\
\hline 20030324 & 1 ECL 3 & $22: 02: 36$ & TP, PV & d3o.dat & yes & 1 & III & I & (not recorded) \\
\hline 20030326 & 2 ECL 1 & 20:42:00 & TP, PV & d3q.dat & yes & 1 & I & II & passing clouds \\
\hline 20030402 & 2 ECL 1 & 22:59:05 & TP, PV & \multicolumn{6}{|c|}{ no lightcurve: clouds } \\
\hline 20030411 & 3 ECL 2 & 21:03:03 & TP, PL & d4b.dat & yes & 1 & II & I & thick cirrus + moon 1 st quarter \\
\hline 20030411 & 4 ECL 1 & $21: 26: 45$ & TP, PL & d4b.dat & yes & 1 & I & II & thick cirrus + moon 1 st quarter \\
\hline
\end{tabular}

Table 2. Individual notes on phenomena.

\begin{tabular}{|c|c|c|}
\hline 19970919 & 4 OCC 3 & $\begin{array}{l}\text { Due to the large distance between the monitored phenomenon and the reference object there are some } \\
\text { sudden jumps in the light curve that are probably due to clouds. }\end{array}$ \\
\hline 19970925 & 1 OCC 3 & $\begin{array}{l}\text { For part of the observation the sky background could not be modeled. This part of the light curve has been } \\
\text { removed. There is no obvious explanation for the rise of the light curve in the beginning and the subsequent } \\
\text { decrease. The difference between the satellites involved in the phenomenon and the reference satellite is } \\
\text { one magnitude larger than expected. }\end{array}$ \\
\hline 19970925 & 1 ECL 3 & Very ba \\
\hline 19970929 & 3 OCC 2 & $\begin{array}{l}\text { For several parts of the observation the sky background could not be modeled. These parts of the light } \\
\text { curve have been removed. }\end{array}$ \\
\hline 19971007 & 4 OCC 1 & $\begin{array}{l}\text { Due to clouds the timing of the phenomenon is lost after 19:41:52. Around 19:34:44 and 19:39:14 due to } \\
\text { clouds the satellites almost disappeared. The most problematic periods were removed from the light curve. } \\
\text { Very variable transparency of the sky. }\end{array}$ \\
\hline 19971125 & OCC 1 & Jupiter and the satellites remained in a hole between the clouds, until they disappeared at 18:46. \\
\hline 20030109 & 3 OCC 1 & $\begin{array}{l}\text { The proximity of Jupiter caused some blooming on some of the images. There is an unexplained quasi- } \\
\text { periodicity in the light curve (period } \sim 1.2 \mathrm{~min} \text { ). The flashing mechanism did not work, therefore timings } \\
\text { were recorded by hand. }\end{array}$ \\
\hline 20030203 & 2 OCC 3 and 2 ECL 3 & One light curve for two phenomena. Both phenomena overlap. \\
\hline 20030227 & $1 \mathrm{OCC} 4$ & $\begin{array}{l}\text { Flashes were generated only after the phenomenon, such that timings are extrapolations rather than inter- } \\
\text { polations. However, the error in the timings should be no larger than } 0.2 \mathrm{~s} \text {. }\end{array}$ \\
\hline 20030315 & 3 ECL 4 & $\begin{array}{l}\text { Part of the light curve had to be removed because the satellite moved too close to the border of the window. } \\
\text { Slower acquisition rate. }\end{array}$ \\
\hline 20030318 & 3 & nolo lo \\
\hline 20030326 & 2 ECL 1 & $\begin{array}{l}\text { Large parts of phenomenon lost in clouds. Timings may be systematically off by } 0.1 \mathrm{~s} \text {, because the first } \\
\text { image of each object was not visible. }\end{array}$ \\
\hline 20030411 & 3 ECL 2 & $\begin{array}{l}\text { Two phenomena in one light curve. The satellite involved in the second phenomenon was used as reference } \\
\text { for the first phenomenon and vice versa. }\end{array}$ \\
\hline
\end{tabular}


These data have been transmitted to Jean-Eudes Arlot for further processing and to be analysed together with all the data obtained from all participating observatories.

It is puzzling that for occulations the light curve invariably increases before the phenomenon, and decreases again afterwards. There is no such behaviour for eclipses, where the light curve is flat outside the phenomenon. At first we thought this was due to the satellites moving apart and thus filling a larger box after the phenomenon while the aperture of the "aperture photometry" remained constant, causing an increasing part of the light to fall outside the box. However, in the second version of the reduction, where the pixels containing a satellite image were determined automatically (such that the "size of the aperture" was adapted automatically), the phenomenon is still as prominent. We have no explanation for this phenomenon.
Acknowledgements. The authors would like to thank the technical staff of the Royal Observatory for rapid problem-solving, so that the telescope was available in time to observe some of the phenomena. The authors would also like to thank Patricia Lampens for her participation in the observations of one of the phenomena. We want to thank the referee for useful comments.

\section{References}

Aksnes, K. 1997, in Proc. of the 5th workshop PHEMU97, ed. J.-E. Arlot, \& C. Blanco, 17

Arlot, J.-E. 1997, in Proc. of the 5th workshop PHEMU97, ed. J.-E. Arlot, \& C. Blanco, 59 